\title{
Adding chimeric antigen receptor-induced killer cells to the medical oncology shelf
}

\author{
Brigett D. Brandjes and Marco L. Davila \\ Blood \& Marrow Transplantation and Cellular Immunotherapy, H. Lee Moffitt Cancer Center and Research Institute, Tampa, Florida, USA.
}

\begin{abstract}
With the approval of CD19-targeted chimeric antigen receptor (CAR) T cells for the treatment of B cell malignancies, clinicians have gained valuable insights into the power and challenges of cellular therapies. In this issue of the $J C l$, Maluski et al. showed that a CAR containing a CD28 costimulatory domain drives progeny differentiation to resemble that of NK cells, which have the potential for an off-the-shelf cell therapy. These CAR-induced killer (CARiK) cells displayed potent antitumor function and killed across the MHC barrier in vivo. After performing in vitro and in vivo mouse studies, the authors also successfully differentiated human umbilical cord blood-derived progenitor cells into CARiK cells. These unique cells may address some of the current challenges associated with first-generation CARs, such as prolonged production that requires patients to wait weeks for infusion. We believe this innovative progenitor gene-engineered lymphoid system has the potential for clinical translation.
\end{abstract}

\section{Gene-engineered chimeric} antigen receptor cell therapies It has been two years since the FDA approved gene-engineered chimeric antigen receptor (CAR) $\mathrm{T}$ cell therapy for the treatment of $\mathrm{B}$ cell acute lymphoblastic leukemia and diffuse large B cell lymphoma $(1,2)$. Our institution has treated more than 100 patients with CAR T cells as the standard of care, and we appreciate both the opportunities and the challenges that this therapy offers (3). The ability to induce durable remissions in patients with chemotherapy-refractory leukemias or lymphomas is highly valuable. However, for some patients, this treatment is complicated by manufacturing time, production failures, disease progression, and the need for bridging chemotherapy. These challenges are unique in medical oncology because they are inherent to a therapeutic model that requires cell collection, gene engineering, cell expansion, and product-release studies. If the treatment remains relegated to the small number of academic medical centers currently offering CAR T cells, these challenges may limit the extent of therapeutic success.

Academic and industry efforts are being directed to developing off-the-shelf gene-engineered cell therapies, which could mitigate these challenges. Features of an ideal off-the-shelf therapy would include rapid availability, reduced cost, persistence, efficacy and/or toxicity equivalent if not superior to its autologous competitor, and absence of genetic mutations that may compromise the long-term health of recipients (2). The main distinctions between off-the-shelf adoptive cell therapies for cancer arise from the cell sources. Examples include primary cells, derived

Related Article: p. 5108

Conflict of interest: MLD receives research support from Atara Biotherapeutics, Celgene, and Precigen and has licensed CAR technologies to Atara Biotherapeutics. MLD has stock options in Precision BioSciences and Adaptive Biotechnologies.

Copyright: (c) 2019, American Society for Clinical Investigation.

Reference information: J Clin Invest. 2019;129(12):5077-5078. https://doi.org/10.1172/JCI132536.

from unrelated, healthy donor cells that are subsequently gene modified ex vivo, or primary cells transformed to a long-lived state, such as a cell line or pluripotent stem cell, also gene modified ex vivo.

Off-the-shelf cell therapies derived from allogeneic donors carry the risk of toxicity due to alloreactivity. Some methods to prevent this incompatibility use cells lacking alloreactive $\mathrm{T}$ cell receptors (TCR), such as $\gamma \delta$ T cells, viral-specific T cells, or NK cells, as host for the CAR $(4,5)$. Since such a system requires collecting scarce cells from the blood, significant ex vivo expansion is necessary, which thus extends production time. While production time increases would not delay patient treatment, lengthy ex vivo cell culture is associated with terminal differentiation and exhaustion (6). Another method requiring two genetic manipulations to delete and replace the TCR with a CAR (2) is not $100 \%$ efficacious, requires enrichment and selection of TCR-negative cells, and sometimes results in multiple rounds of TCR deletion. Genome editing may also generate off-target mutations, which could lead to malignant transformation of the cell product. Even "safe" lentiviral modification of peripheral $\mathrm{T}$ cells can integrate into genetic loci and dramatically change the $\mathrm{T}$ cell phenotype (7). Additionally, genetic manipulations present in a single production will be infused in multiple recipients. Consequently, a single deleterious genome editing and/or viral transduction could result in complications in many patients. Among the multiple genome-editing systems, there are likely different off-target mutation rates and variable risks for these toxicities, so we must wait for clinical reporting with each respective product.

While these products use healthy donor cells for gene engineering, others use cell lines such as cytotoxic NK cell lines or induced pluripotent stem (iPS) cells (8). iPS cells are unique, as they allow for the expansion of differentiated CAR-expressing lymphoid cells from a cryopreserved 
stem cell that can be used for multiple productions $(9,10)$. Both iPS cells and other cell lines allow extensive genetic manipulations to enhance function and reduce toxicities. However, similarly to genome-edited products, they share the risk of imposing unsafe genetic changes. Even the process required to derive iPS cells could compromise long-term safety. While clinical evaluations of off-the-shelf products are still early, the main therapeutic concern is shortened persistence, probably due to host rejection (11).

\section{Advancing adoptive cell therapies}

Maluski and colleagues (12) differentiated hematopoietic stem cells (HSC) that were gene targeted with a CAR construct into lymphoid cells ex vivo. This ex vivo differentiation platform used OP9-DL1 cells, which have been employed in prior cases for progenitor development and even gene modification (13, 14). However, Maluski et al. used the system to significantly advance adoptive cell therapy and showed the potential for clinical translation. The authors used a CAR construct that included a CD28 costimulatory domain to drive progeny differentiation to an NK-like phenotype, which they described as CARinduced killer (CARiK) cells (12).

First-generation CARs, which included only $\mathrm{CD} 3 \zeta$ activation domains, were inferior at driving differentiation toward CARiK and had poor antitumor function. Maluski et al. demonstrated that CARiK are not only efficacious killers, but kill across MHC barriers in vivo. This function is necessary for an offthe-shelf cell therapy used in the context of an allogeneic stem cell transplantation (alloSCT). In addition, the authors showed that regulation of CAR expression during progenitor cell differentiation directed the cells to T or NK cells. Controlling the cell fate may allow for production of CARiK or CAR T cells depending on whether either cell is better for treating a specific tumor type. Finally, Maluski and authors differentiated human progenitor cells into CARiK, confirming the potential for clinical translation (12).

A major advantage of the CARiK platform compared with genome-editing products includes the reduced chance for deleterious off-target mutations. However, insertional oncogenesis remains a concern, since retrovirus gene transfer into hematopoietic progenitors has resulted in T cell leukemia (15). An advantage of using progenitor cells during alloSCT is the lower likelihood for host rejection, which is a major reason for shortened persistence of off-the-shelf products in current use (16). In fact, to mitigate host rejection, some conditioning regimens include alemtuzumab, which can worsen infectious complications (16). Another advantage to differentiating progenitor cells during alloSCT is that it circumvents or limits problems with alloreactivity and graft-versus-host disease. However, on the flip side, alloSCT still results in problematic morbidities and mortalities and remains a treatment reserved for patients with few options

Like all innovative work, the Maluski report (12) stimulates follow-up questions. How would incorporating the costimulatory domain 4-1BB into the CAR construct affect progenitor differentiation? How can we optimize CARiK when they are essentially a cell subset that has never been described heretofore? Can immune receptor-based activation motifs (ITAMs), costimulatory domains, or timing of CAR expression modulate this platform to create a wide variety of immune effector CAR cells? And how might this off-the-shelf product compare with other cell products? We are hopeful that the findings presented by Maluski et al. (12) will ultimately lead to off-theshelf products that support clinical outcomes equivalent to the autologous CAR T cell products currently approved, but without the wait (or cost) - a breakthrough that patients and clinicians would welcome.

\section{Acknowledgments}

We are grateful to the nurses, pharmacy, and staff that make up the Moffitt Immune Cell Therapy (ICE T) service.

Address correspondence to: Marco L. Davila, Blood \& Marrow Transplantation and Cellular Immunotherapy, H. Lee Moffitt Cancer Center and Research Insti- tute, 12902 USF Magnolia Drive, Tampa, Florida 33612, USA. Phone: 813.745.7202; Email: marco.davila@moffitt.org.

1. Neelapu SS, et al. Axicabtagene ciloleucel CAR T-cell therapy in refractory large B-cell lymphoma. N Engl J Med. 2017;377(26):2531-2544.

2. Maude SL, et al. Tisagenlecleucel in children and young adults with B-cell lymphoblastic leukemia. N Engl J Med. 2018;378(5):439-448.

3. Locke FL, Anasetti C, Moffitt Immunotherapy Working Group the Immune Cell Therapy (ICET) Program. Transplanters drive CARs to the clinic by brewing ICE-T: the Moffitt roadmap. J Immunother Cancer. 2017;5(1):59.

4. Lupo KB, Matosevic S. Natural killer cells as allogeneic effectors in adoptive cancer immunotherapy. Cancers (Basel). 2019;11(6):E769.

5. Capsomidis A, et al. Chimeric antigen receptor-engineered human gamma delta T cells: enhanced cytotoxicity with retention of cross presentation. Mol Ther. 2018;26(2):354-365.

6. Chacon JA, et al. Co-stimulation through 4-1BB/ CD137 improves the expansion and function of CD8(+) melanoma tumor-infiltrating lymphocytes for adoptive T-cell therapy. PLOS ONE. 2013;8(4):e60031.

7. Fraietta JA, et al. Disruption of TET2 promotes the therapeutic efficacy of CD19-targeted T cells. Nature. 2018;558(7709):307-312.

8. Tang $X$, et al. First-in-man clinical trial of CAR NK-92 cells: safety test of CD33-CAR NK-92 cells in patients with relapsed and refractory acute myeloid leukemia. Am J Cancer Res. 2018;8(6):1083-1089.

9. Themeli M, Rivière I, Sadelain M. New cell sources for $\mathrm{T}$ cell engineering and adoptive immunotherapy. Cell Stem Cell. 2015;16(4):357-366.

10. Themeli M, et al. Generation of tumor-targeted human Tlymphocytes from induced pluripotent stem cells for cancer therapy. Nat Biotechnol. 2013;31(10):928-933.

11. Graham C, Jozwik A, Pepper A, Benjamin R. Allogeneic CAR-T cells: more than ease of access? Cells. 2018;7(10):E155.

12. Maluski M, et al. Chimeric antigen receptorinduced BCL11B suppression propagates NK-like cell development. JClin Invest. 2019;129(12):5108-5122.

13. Zakrzewski JL, et al. Tumor immunotherapy across MHC barriers using allogeneic T-cell precursors. Nat Biotechnol. 2008;26(4):453-461.

14. Zakrzewski JL, et al. Adoptive transfer of T-cell precursors enhances $\mathrm{T}$-cell reconstitution after allogeneic hematopoietic stem cell transplantation. Nat Med. 2006;12(9):1039-1047.

15. Hacein-Bey-Abina S, et al. LMO2-associated clonal $\mathrm{T}$ cell proliferation in two patients after gene therapy for SCID-X1. Science. 2003;302(5644):415-419.

16. Benjamin R. CD56 targeted chimeric antigen receptors for immunotherapy of multiple myeloma. Cancer Res. 2012;72(8 Suppl):3499. 